\section{A evolucão do Congresso Brasileiro de Atividáade Física e Saúde. Copo meio cheio ou meio vazio?}

\section{The evolution of the Brazilian Congress on Physical Activity and Health. Glass half full or half empty?}

Tais Taiana Sarabia ${ }^{12}$

Mariana Silva dos Reis ${ }^{12}$

Adriano Vescovi ${ }^{3}$

Thiago Hérick de Sá

Leandro Fórnias Machado de Rezende

\section{RESUMO}

O objetivo do presente estudo foi resgatar a memória do Congresso Brasileiro de Atividade Física e Saúde (CBAFS) e descrever a evolução da participação no evento segundo localização geográfica do estado de origem do primeiro autor dos resumos, eixos de pesquisa, e tipo de estudo dos resumos apresentados. A pesquisa foi realizada nos anais das nove edições do CBAFS. Buscou-se identificar o estado (convidados nacionais) e país (convidados internacionais) de origem dos palestrantes, estado de origem do primeiro autor, delineamento de estudo e eixos de pesquisa em atividade física e saúde (em que a atividade física e/ou o comportamento sedentário são exposição ou desfecho do estudo). Os dados foram apresentados em frequências absolutas, relativas e taxas brutas por milhão de habitantes. Em todos os anos foi observada uma predominância de palestrantes e de resumos da região Sul do país, seguido das regiões Sudeste e Nordeste. Quanto aos palestrantes internacionais, houve participação da América do Norte (EUA, Canadá), Europa (Portugal, França, Finlândia e Dinamarca) e Colômbia. No conteúdo dos resumos há predominância de delineamentos transversais sobre as consequências da atividade física/comportamento sedentário na saúde, determinantes da prática de atividade física e estudos descritivos sobre nível/tendência de atividade física. $\mathrm{O}$ eixo de intervenções para aumento dos níveis de atividade física e diminuição do comportamento sedentário permanece incipiente. Conclui-se que há grande disparidade geográfica em relação aos congressistas e dificuldades em traduzir as evidências em aumento nos níveis de atividade física da população. Entretanto, tem sido observada uma melhora em relação a representação geográfica e crescente número de participantes.

\section{PALAVRAS-CHAVE}

Atividade motora; Localizações geográficas; Congressos.

\section{ABSTRACT}

The aim of this study was to unveil the history of the Brazilian Congress of Physical Activity and Health (CBAFS) and describe the evolution of the event according to participants' geographical location of the affiliation, research topics, and type of study presented in abstracts. The survey was conducted in annals of all nine CBAFS editions. We sought to identify the state (national guests) and country (international guests) of speakers' origin, as well as first autbor state, study design and research lines in physical activity and health (where physical activity and/or sedentary behavior are exhibition or study endpoint). The data was presented as absolute frequency, relative frequency and crude rates per million inhabitants. In all years a predominance of speakers and abstracts of the Southern region, followed by the Southeast and Northeast regions was observed. Regarding international speakers there was participation from North America (USA, Canada), Europe (Portugal, France, Finland and Denmark) and Colombia. For the content of the abstracts there was a predominance of cross-sectional designs on the consequences of physical activity/sedentary behavior, determinants of physical activity and descriptive studies on levels/trends of physical activity. The topic of interventions to increase levels of physical activity and decrease sedentary behavior remained incipient. We Concluded that there are still great geographical disparity in relation to congressmen and difficulties in translating evidence into increased levels of physical activity. However, an improvement has been observed in relation to geographical representation and growing number of participants'.

KEYWORDS

Motor activity; Geographic locations; Congresses.
Rev Bras Ativ Fis Saúde p. 627-635

DO

http://dx.doi.org/10.12820/rbafs.v.19n5p627

1 Universidade Federal do Paraná, Programa de Pós-graduação em Educação Física, Curitibal $P R$ - Brasil.

2 Grupo de Pesquisa em Atividade Física e Qualidade de Vida - GPAQ - PUCPR.

3 Universidade Federal do Espírito Santo, Programa de Pós-graduação em Educação Física, Vitória/ES-Brasil.

4 Faculdade de Saúde Pública - Universidade de São Paulo, Departamento de Nutrição, São Paulo/ SP - Brasil

5 Faculdade de Medicina da Universidade de São Paulo, Departamento de Medicina Preventiva, São Paulo/SP - Brasil. 


\section{INTRODUCCÃO}

Desde 1985 a Universidade Federal de Santa Catarina (UFSC) promovia o Simpósio de Pesquisa em Educação Física. A importante expressividade deste simpósio resultou em sua ampliação e na criação de um novo nome: Congresso Brasileiro de Atividade Física e Saúde (CBAFS). O CBAFS foi promovido pela primeira vez em 1997 pela USFC, através do Núcleo de Pesquisa em Atividade Física e Saúde (NuPAF) e do Programa de Pós-Graduação em Educação Física desta mesma instituição ${ }^{1}$. Desde sua origem o CBAFS ocorre de forma bianual. Em 2001 foi realizada sua primeira edição isolada, deixando de ocorrer em paralelo com o Simpósio de Pesquisa em Educação Física (que ficou destinado aos anos pares a partir de 2002) e passou a ser itinerante a partir de sua sétima edição (2009).

O CBAFS nasceu com o objetivo de congregar pesquisadores, profissionais e estudantes em torno da discussão sobre evidências científicas e aplicações da atividade física para a saúde, visando proporcionar aos seus participantes a perspectiva de ampliação de conhecimentos e intercâmbio de experiências. Desde o início e ao longo das edições, esta missão pode ser observada nos relatos do professor Markus V. Nahas, idealizador e presidente de cinco das nove edições:

"[...] o CBAFS veio para ficar como um dos mais importantes fóruns de discussão na área no Brasil[...] buscar-se-á promover um ambiente de interação entre os pesquisadores da área, como também demonstrar para a sociedade, através de pesquisas científicas, a importância da associação entre a atividade física, aptidão física e saúde na prevenção de diversas doenças consideradas problemas de saúde pública no Brasil e em outros países”2.

“" [...] o CBAFS se propõe a dar continuidade na promoção de um evento que tem proporcionado intercambio significativo entre profissionais e estudantes da área, e permitindo a divulgação dos estudos desenvolvidos”3.

O sucesso de todo o trabalho com o CBAFS frutificou na criação da Sociedade Brasileira de Atividade Física e Saúde (SBAFS) em 16 de novembro de 2007. No mesmo ano, a Revista Brasileira de Atividade Física e Saúde (RBAFS), criada pela Associação dos Professores de Educação Física de Londrina, foi cedida à SBAFS, tornando-se o periódico oficial da sociedade, conforme relato do professor Markus V. Nahas:

“[...] há um grande movimento para a criação de uma Sociedade Brasileira de Atividade Física e Saúde, em decorrência do crescimento da área e da sentida necessidade de uma entidade aglutinadora das iniciativas acadêmicas [...] . Temos hoje uma plêiade de novos cientistas [...] desenvolvendo pesquisas relevantes em quantidade e qualidade que permitem, também, idealizarmos a criação de um periódico científico vinculado a sociedade que se pretende criar e lançar durante o $6^{\circ}$ CBAFS"4.

Uma das solicitações do presente para almejar um futuro ainda mais promissor para o CBAFS é entender seu processo de evolução, principalmente em relação a representatividade nacional. Espera-se que um congresso dito 
como brasileiro congregue pesquisadores, profissionais e alunos das diferentes regiões da nação. Outra importante reflexão diz respeito ao avanço das pesquisas apresentadas no congresso. Será que o conhecimento da área de atividade física e saúde no Brasil está evoluindo? Em que proporção $0^{5}$.

Para que o avanço do conhecimento aconteça, a pesquisa precisa seguir seu ciclo: conhecer os níveis de atividade física e seus fatores determinantes, conhecer as consequências da atividade físicas para a saúde, realizar estudos confirmatórios e desenvolver intervenções com o objetivo de influenciar as realidades encontradas. Uma análise detalhada dos eixos de pesquisa combinada com os tipos de estudo dos resumos apresentados no CBAFS permitirá conhecer o desenvolvimento e contribuir para o amadurecimento da área de atividade física e saúde no Brasil.

O presente artigo tem como objetivo descrever a evolução da participação no evento segundo localização geográfica dos participantes, eixos de pesquisa, e tipo de estudo dos resumos apresentados no CBAFS.

\section{MÉTODOS}

Trata-se de uma série temporal de cortes transversais, baseada em busca realizada em março de 2014 nos anais das nove edições já realizadas do CBAFS para identificação dos palestrantes convidados e dos resumos publicados. A leitura dos títulos e resumos foi realizada por um único revisor com o objetivo de classificar os resumos segundo localização (estado de origem do primeiro autor nos resumos nacionais, e país para os internacionais), tipos de estudo e eixos de pesquisa ${ }^{6-14}$. Ainda foi extraída a localização dos palestrantes nacionais e internacionais.

Em relação aos tipos de estudo, os resumos foram classificados em estudo de caso, série de casos, transversal, caso controle, coorte, experimental, metodológico e outros, como base na literatura clínico-epidemiológica ${ }^{15}$ Adicionalmente, os resumos foram classificados segundo o eixo de pesquisa em Atividade Física e Saúde ${ }^{16}$. Para tanto, foram considerados somente estudos que incluíram a atividade física e/ou o comportamento sedentário como exposição ou desfecho. Seguindo esse critério, os estudos foram classificados em:

1) Nível de atividade física e tendência temporal: prevalência de atividade física;

2) Determinantes da prática de atividade física: associação de variáveis sociodemográficas, variáveis ambientais e genéticas com a prática de atividade física;

3) Consequências da prática de atividade física na saúde: benefícios da atividade física para a saúde, estudos que tendem a esclarecer dose-resposta;

4) Intervenções para aumento do nível de atividade física: estudos de acompanhamento;

5) Ações e planejamento regional, nacional e global em atividade física e saúde;

6) Metodológicos: estudos com objetivo de avaliar a validade e/ou reprodutibilidade de testes e medidas;

7) Outros: estudos que não incluíam atividade física ou comportamento sedentário como exposição ou desfecho, bem como resumos na forma de comentários, reflexões e textos de advocacy, incluindo estudos sobre a aptidão física e desenvolvimento motor. 
A descrição da localização dos palestrantes nacionais e internacionais foi apresentada em frequências absolutas e relativas. O número de resumos de todos os congressos, segundo os estados da federação, foi apresentado em frequências absolutas e taxas brutas por milhão de habitantes. Foi considerada como população de referência as projeções do IBGE para 2013 a partir do Censo 2010. Estimativas populacionais para os municípios brasileiros em 01.07.2013. (http://www.ibge.gov.br/home/estatistica/populacao/estimativa2013/). A frequência absoluta dos resumos também foi apresentada segundo eixo de pesquisa e tipo de estudo.

\section{RESULTADOS}

Em 2013, o CBAFS chegou em sua IX edição. Durante a sua história, o CBAFS aconteceu predominantemente na região Sul, sempre no mês de novembro. Informações sobre o local, temática, presidentes e apoiadores das nove edições estão sumarizados no Quadro 1.

QUADRO 1 - Edições e temáticas do CBAFS.

\begin{tabular}{|c|c|c|c|c|}
\hline Data & Local & Temática & Presidente & $\begin{array}{l}\text { Órgãos financiadores } \\
\text { e/ou apoiadores }\end{array}$ \\
\hline $\begin{array}{l}5 \text { a } 7 \text { de } \\
\text { novembro } \\
1997\end{array}$ & & $\begin{array}{l}\text { Atividade Física, Aptidão Física e } \\
\text { Saúde: Avanços Científicos e Perspectivas } \\
\text { para a Educação Física }\end{array}$ & Markus V. Nahas & CNPQ, CAPES, INDESP, UFSC \\
\hline $\begin{array}{l}24 \text { a } 26 \text { de } \\
\text { novembro } \\
1999\end{array}$ & & $\begin{array}{l}\text { Promoção de um Estilo de Vida Ativo: da } \\
\text { Criança ao Idoso }\end{array}$ & Maria F. S. Duarte & CNPQ, CAPES, SESI \\
\hline $\begin{array}{l}15 \text { a } 17 \text { de } \\
\text { novembro } \\
2001\end{array}$ & & $\begin{array}{l}\text { Promoção de Atividade Física: da Pesquisa } \\
\text { à Ação }\end{array}$ & Markus V. Nahas & CNPQ, CAPES, SESI \\
\hline $\begin{array}{l}20 \text { a } 22 \text { de } \\
\text { novembro } \\
2003\end{array}$ & $\begin{array}{l}\text { Florianópolis } \\
\text { SC }\end{array}$ & $\begin{array}{l}\text { Atividade Física Saúde e Bem-estar: na } \\
\text { Escola, na Empresa e na Comunidade }\end{array}$ & Markus V. Nahas & SESI \\
\hline $\begin{array}{l}17 \text { a } 19 \text { de } \\
\text { novembro } \\
2005\end{array}$ & & Qualidade de Vida, Atividade Física e Lazer & Markus V. Nahas & $\begin{array}{l}\text { SESI, CAPES, UFSC, EMCATUR, CREF, } \\
\text { Jamine Alimentos integrais }\end{array}$ \\
\hline $\begin{array}{l}15 \text { a } 17 \text { de } \\
\text { novembro } \\
20007\end{array}$ & & $\begin{array}{l}\text { Interdisciplinaridade na Promoção de } \\
\text { Estilos de Vida Ativos }\end{array}$ & Markus V. Nahas & $\begin{array}{l}\text { SESI, ABQV - Associação Brasileira } \\
\text { de Qualidade } \\
\text { de Vida Ministério da Saúde, ILSI- } \\
\text { Brasil }\end{array}$ \\
\hline $\begin{array}{l}12 \text { a } 14 \text { de } \\
\text { novembro } 2009\end{array}$ & $\begin{array}{l}\text { Porto de } \\
\text { Galinhas } \\
\text { PE }\end{array}$ & $\begin{array}{l}\text { Atividade Física no Sistema Único de } \\
\text { Saúde: da Atenção Básica às Ações } \\
\text { Intersetoriais }\end{array}$ & Mauro V. Barros & $\begin{array}{l}\text { Ministério da Saúde, SESI, CAPES, } \\
\text { FACEPE, CNPQ, Secretaria das } \\
\text { Cidades do Estado de Pernambuco, } \\
\text { Prefeitura da Cidade de Recife e SESC }\end{array}$ \\
\hline $\begin{array}{l}9 \text { a } 12 \text { de } \\
\text { novembro } 2011\end{array}$ & $\begin{array}{l}\text { Gramado } \\
\text { RS }\end{array}$ & $\begin{array}{l}\text { Atividade Física e Saúde: da Concepção ao } \\
\text { Envelhecimento }\end{array}$ & Pedro C. Hallal & $\begin{array}{l}\text { Ministério da saúde, CAPES, SESI, } \\
\text { SESC }\end{array}$ \\
\hline $\begin{array}{l}13 \text { a } 16 \text { de } \\
\text { novembro } 2013\end{array}$ & $\begin{array}{l}\text { Curitiba } \\
\text { PR }\end{array}$ & Pessoas Ativas, Cidades Saudáveis & Rodrigo S. Reis & $\begin{array}{l}\text { SESI, Fundação Araucária, PGEDF, } \\
\text { UFPR, CAPES, SUS, Ministério da } \\
\text { Saúde } \\
\text { Governo Federal Brasil }\end{array}$ \\
\hline
\end{tabular}

Em todos os anos foi observada uma predominância de palestrantes da região Sul do país (33\% a $75 \%$ dos palestrantes), com contribuição importante do estado de Santa Catarina, seguido do Rio Grande do Sul e do Paraná (Figura 1). A segunda e terceira maior participação de palestrantes vem das regi- 
ões Sudeste e Nordeste, respectivamente. O Centro-Oeste esteve presente em seis edições do CBAFS, com a participação de um a três palestrantes, sempre do Distrito Federal, enquanto a região Norte ainda não teve um representante entre os palestrantes. Entre todas as edições do CBAFS, a edição de 2009 (Porto de Galinhas) foi a única sediada fora da região Sul, além de contar com uma maior distribuição dos palestrantes nacionais entre as regiões do Brasil.

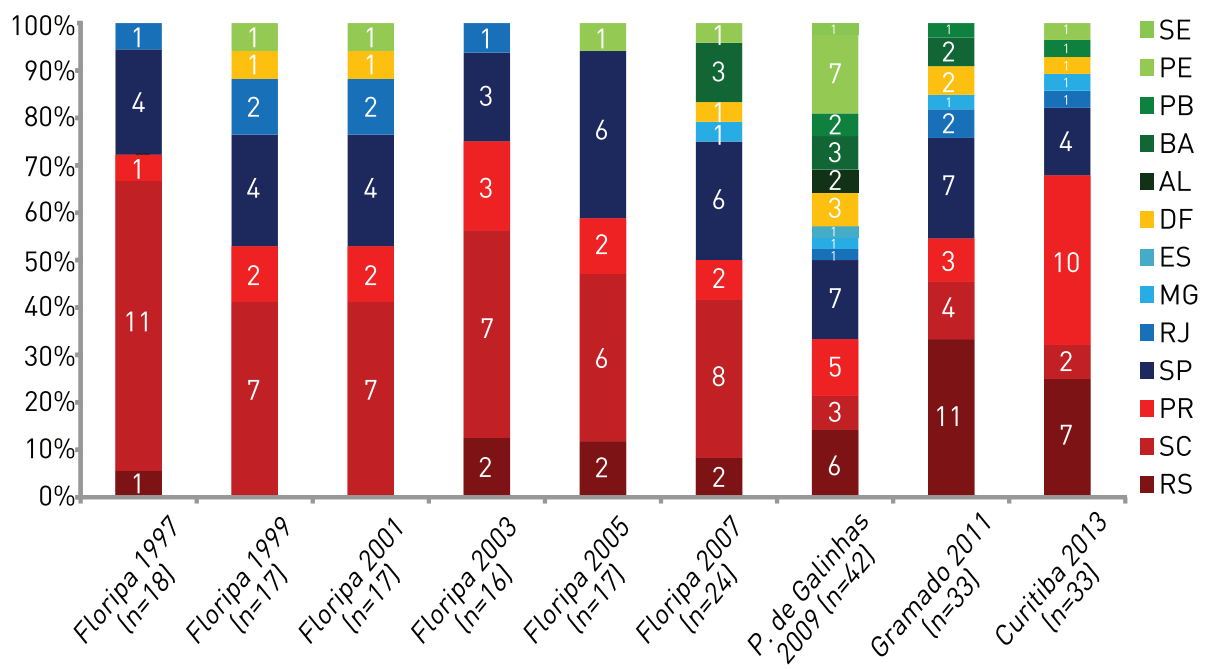

FIGURA 1 - Distribuição dos palestrantes nacionais no Congresso Brasileiros de Atividade Física e Saúde segundo estados da federação. Pelotas-RS, 2014.

Quanto aos palestrantes internacionais, as edições do CBAFS trouxeram de dois (Florianópolis - 1997, 1999 e 2003) a oito palestrantes (Curitiba - 2013), representando dois continentes (América do Norte e Europa), com exceção da participação de uma palestrante da América do Sul (Colômbia - Prof ${ }^{a}$. Dr. Olga Lucia Sarmiento) em Curitiba - 2013. Esta foi também a única participação de um palestrante internacional de países de baixa e média renda. A participação de palestrantes dos EUA foi a mais expressiva (33\% a 100\% dos palestrantes internacionais), seguida de Portugal e França, respectivamente (Figura 2).

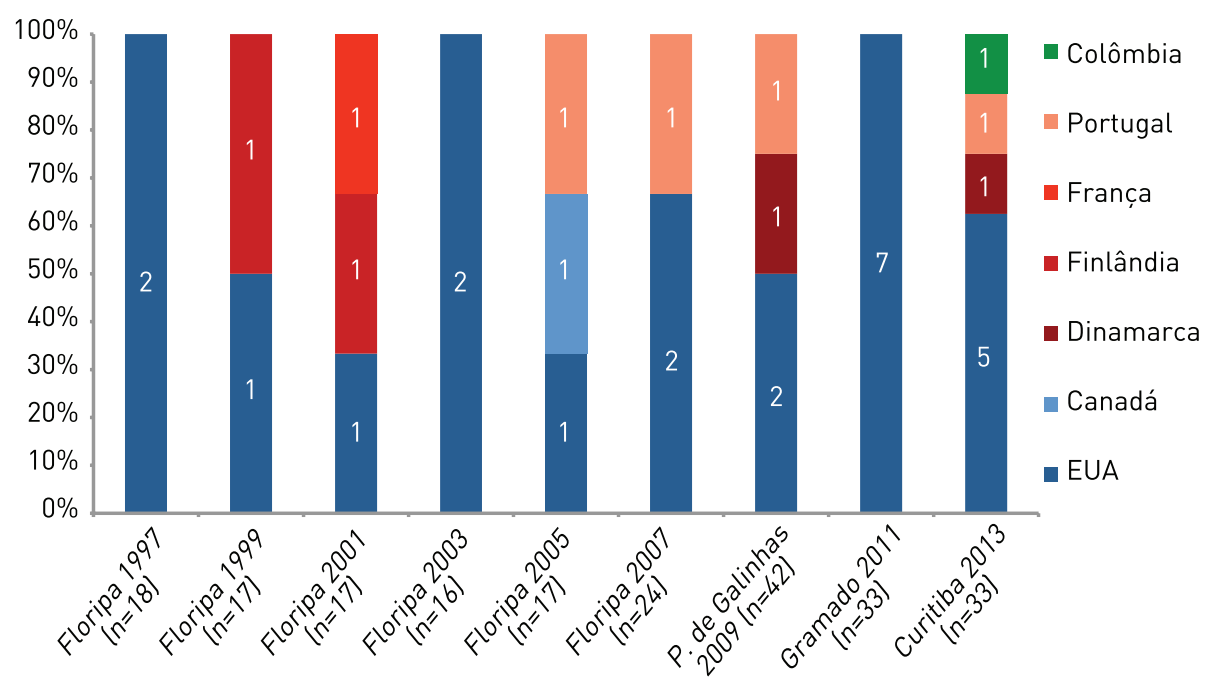

FIGURA 2 - Distribuição dos palestrantes internacionais no Congresso Brasileiros de Atividade Física e Saúde segundo países. Pelotas-RS, 2014. 
Considerando todas as edições do congresso, pode-se observar a participação dos 26 estados e o Distrito Federal na publicação dos resumos (Figura 3). Além disso, houve um aumento do número total de resumos ao longo das edições do CBAFS, com exceção do congresso de 2013 sediado em Curitiba-PR, que limitou o número de inscrições por restrição do espaço físico (Figura 4).

Assim como os palestrantes nacionais, o número de resumos apresentados nas edições do CBAFS apresentou uma alta participação da região Sul, seguida do Sudeste e Nordeste (Figura 3). Quando considerado o número de resumos por milhão de habitantes, destaca-se a grande participação de estados nordestinos como a Paraíba, Pernambuco e Sergipe, e uma menor contribuição de estados do Sudeste como São Paulo, Minas Gerais e Rio de Janeiro (Figura 3), quando comparados com o número absoluto de resumos. Também foi possível observar que a maior parte dos resumos apresentados por representantes de estados do Nordeste e do Norte na história do CBAFS se deu na edição de
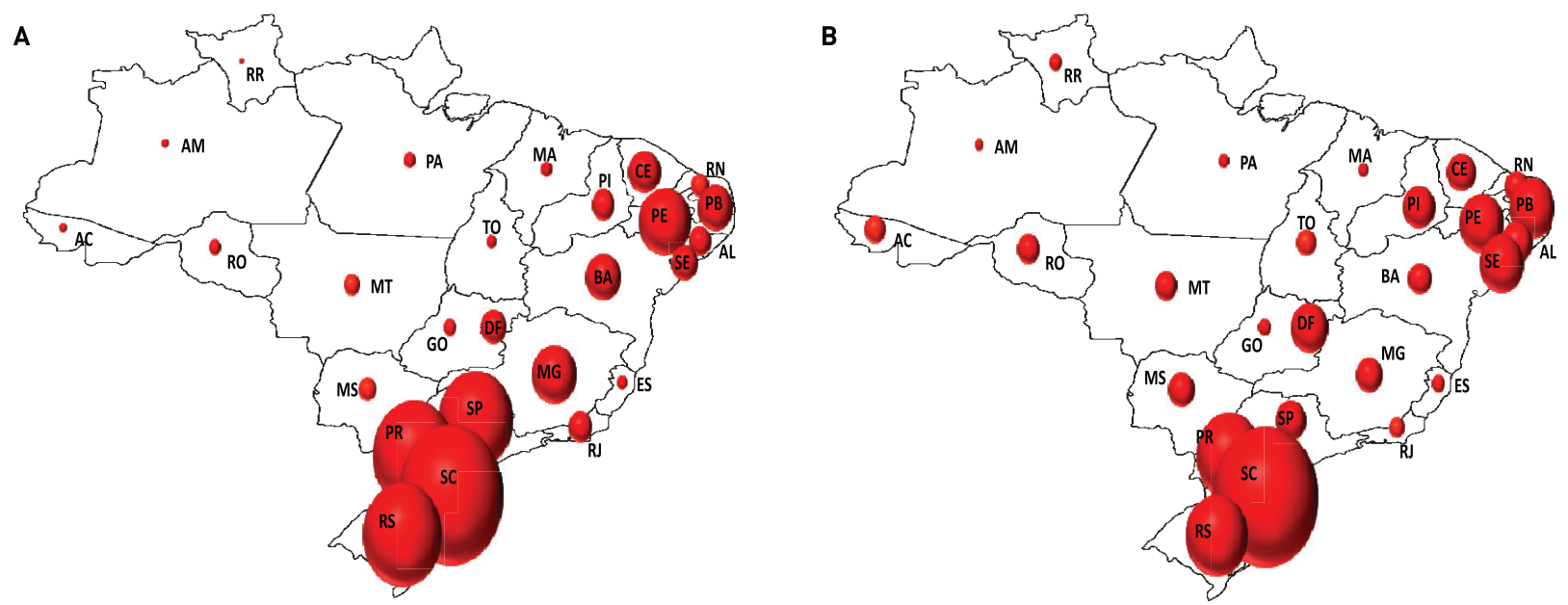

a) Número de resumos por estado; b) Número de resumos por milhão de habitantes

FIGURA 3 - Número de resumos e taxa de resumos (por milhão de habitantes), apresentados no Congresso Brasileiro de Atividade Física e Saúde entre 1997 e 2013 segundo estados da federação.

Porto de Galinhas (2009), e que a participação destas duas regiões nas edições seguintes do congresso (2011 e 2013) foi maior quando comparada com as edições anteriores e 2009 (dados não apresentados).

Em relação ao conteúdo dos resumos apresentados nas edições do CBAFS, em geral, pode-se observar uma predominância de estudos com delineamento transversal (Figura 4) e de eixos de pesquisa como consequências da atividade física/comportamento sedentário na saúde, determinantes da prática de atividade física e estudos descritivos sobre nível de atividade física/tendência. Com o passar das edições do CBAFS, pode-se observar um aumento dos resumos com delineamento experimental (muito relacionado com trabalhos sobre consequências da atividade física para a saúde) e de coorte (Figura 4).

Apesar disso, a grande maioria dos resumos apresentados nas edições mais recentes continuam sendo os de delineamento transversal (Figura 4), e o eixo de intervenções para aumento dos níveis de atividade física e diminuição do comportamento sedentário permanecem incipientes. 

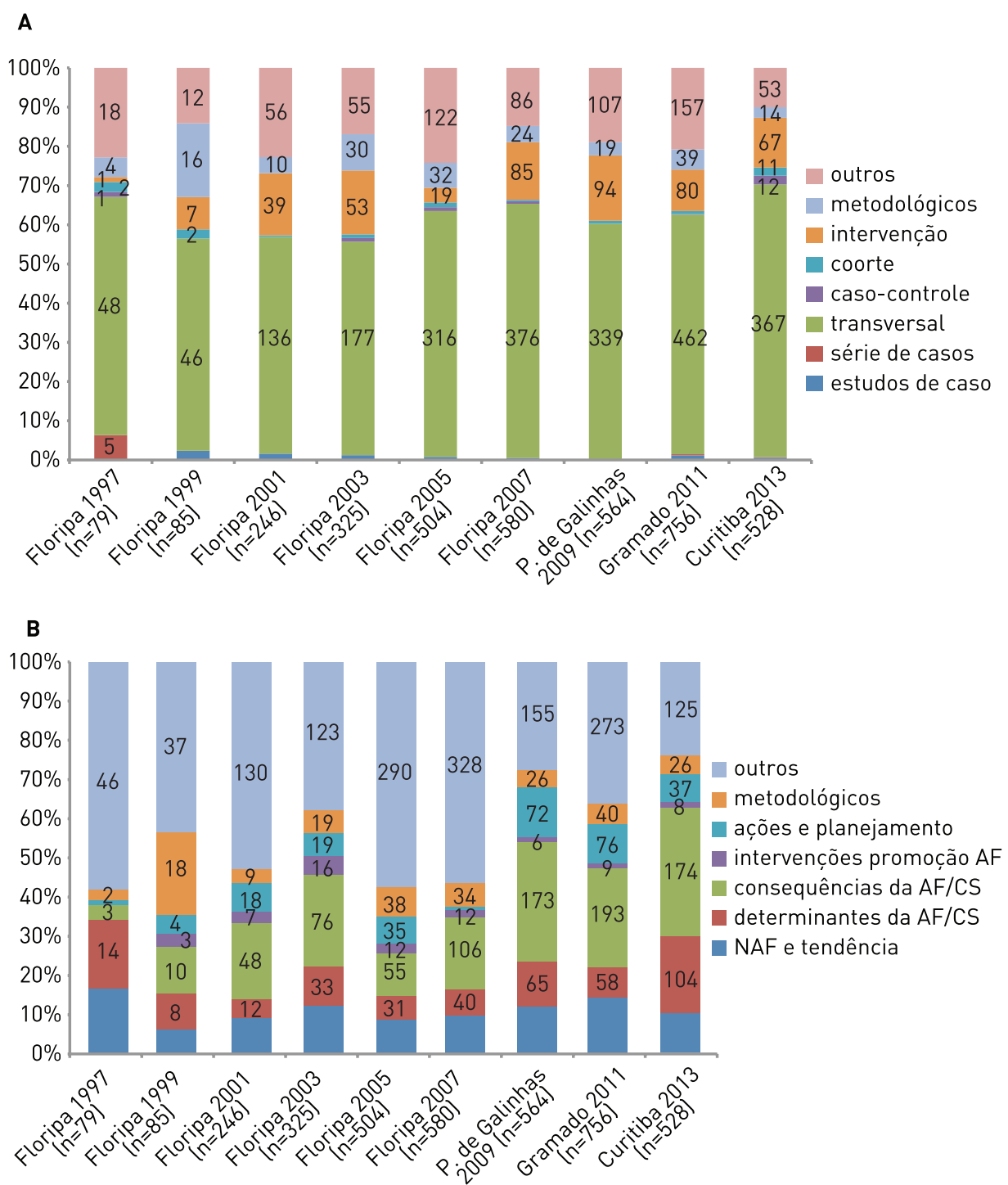

Em caso de sobreposição de legenda, valores absolutos inferiores a 3 foram suprimidos. FIGURA 4 - Distribuição dos resumos no Congresso Brasileiros de Atividade Física e Saúde segundo tipo de estudo.

\section{DISCUSSÃO}

Ao longo desses quase 20 anos de história, o CBAFS tem tido êxito em seu objetivo de congregar e de ser um ponto de encontro para troca de experiências. Isto pode ser observado com o aumento do número total de resumos apresentados no período, bem como a ampliação do número de estados representados no evento. Por outro lado, disparidades geográficas persistem tanto nos resumos quanto nos palestrantes nacionais e internacionais. Além disso, há um aumento na proporção de estudos nos eixos de pesquisa de correlatos/determinantes e consequências da atividade física e comportamento sedentário, e a maior parte dos estudos apresentados sendo de delineamento transversal.

A participação geográfica dos resumos e dos palestrantes nacionais reflete o histórico de evento, centrado na região sul, a existência de mais programas de pós graduação consolidados no centro sul ${ }^{19}$ (a inserção da subárea de Atividade Física e Saúde nos programas de pós-graduação em Educação Física 
no Brasil) e a distribuição populacional do país. Entretanto, permanecem as desigualdades regionais com relação a distribuição geográfica dos resumos quando ajustadas por milhão de habitantes. Essas desigualdades regionais também foram observadas em "publicações brasileiras" sobre atividade física e saúde em revistas internacionais ${ }^{20}$ (A contribuição do Brasil para a pesquisa em atividade física e saúde no cenário internacional). Isto é um indicativo da necessidade de uma maior rede de colaboração dentro da área. Outra possível estratégia para democratizar a participação nos congressos seria levá-lo para outras regiões do Brasil, de modo a facilitar o acesso em termos logísticos e financeiros, além de valorizar as pesquisas desenvolvidas em outros locais. Nesse sentido, parece-nos muito acertada a decisão de levar a próxima edição do congresso para o Maranhão (2015) e a criação dos simpósios regionais de atividade física e saúde.

O fato de haver uma grande concentração de estudos nas áreas de pesquisa de correlatos/determinantes e consequências da atividade física e comportamento sedentário, sobretudo de delineamento transversal, reforçam a necessidade de métodos combinados, perguntas de pesquisa mais ousadas, conectadas com as necessidades atuais e com a participação de diferentes atores (academia, comunidade e políticos), como fora discutido na última edição do CBAFS ${ }^{17}$. Faz-se necessário discussões sobre como tornar as evidências em resultados que possam auxiliar o empoderamento das comunidades, o esclarecimento dos tomadores de decisão, e o estabelecimento de políticas e planos mais efetivos ${ }^{17}$. Essas ações são ainda mais urgentes considerando a pandemia de inatividade física no país, inclusive nas regiões com maior produção científica na área ${ }^{18}$.

Nosso estudo apresenta algumas limitações, sendo que a primeira diz respeito a avaliação de cada resumo ter sido feita por um único revisor. Outra limitação está relacionada com a falta de clareza de alguns resumos, o que tornou a classificação de eixo de pesquisa e tipo de estudo menos precisa.

Como implicações para o acompanhamento da evolução do CBAFS e das pesquisas na área de atividade física e saúde, espera-se que o tipo de delineamento do estudo esteja necessariamente descrito nos métodos, a obrigatoriedade do preenchimento completo das informações pessoais e regionais na plataforma da Sociedade Brasileira de Atividade Física e Saúde, criada em 2013 para a submissão de resumos no congresso, que tem como objetivo facilitar a avaliação dos resumos e também armazenar os anais de todos os congressos subsequentes ao ano de 2013, e principalmente maior rigor nas avaliações dos resumos, cabendo à comissão organizadora determinar os critérios para avaliação.

O copo está meio cheio ou meio vazio? O copo está meio vazio quando tomamos por base a disparidade geográfica na participação no congresso e na incapacidade de traduzir um maior número de evidências de uma região em maiores níveis de atividade física. $\mathrm{O}$ copo está meio cheio quando observamos o aumento do número e a diversidade geográfica de participação, além da consolidação do evento no cenário nacional.

\section{REFERÊNCIAS}

1. Loch MR, Brunetto BC, Rodrigues CG, Nahas MV. A Saúde Pública nos anais do Congresso Brasileiro de Atividade Física e Saúde (1997-2009): revisão sistemática. Rev Bras Ativ Fís Saúde. 2011;16(2):162-67. 
2. NuPAF. Boletim do Nupaf - Núcleo de Pesquisa em Atividade Física e Saúde. CDS/ UFSC. Forianópolis - SC. [online]; 1999. [citado 2014 mar 23]. Disponível em: http:// nupaf.ufsc.br/files/2011/05/Boletim-ano5-n14-Setembro19992.pdf

3. NuPAF. Boletim do Nupaf - Núcleo de Pesquisa em Atividade Física e Saúde. CDS/ UFSC. Florianópolis - SC. [online]; 2001. [citado 2014 mar 23]. Disponível em: http:// nupaf.ufsc.br/files/2011/05/Boletim-ano-16-n40-Setembro-20111.pdf

4. NuPAF. Boletim do Nupaf - Núcleo de Pesquisa em Atividade Física e Saúde. CDS/ UFSC. Florianópolis - SC. [online]; 2007. [citado 2014 mar 23]. Disponível em: http:// nupaf.ufsc.br/files/2011/05/Boletim-ano13-n32-Mar\%C3\%A7o20071.pdf

5. Florindo AA, Rombaldi AJ, Barros MVG. Análise da Revista Brasileira de Atividade Física e Saúde em 2013: O copo está meio cheio ou meio vazio? Rev Bras Ativ Fís Saúde. 2013;18(6):653-54

6. Anais 1 Congresso Brasileiro de Atividade Física e Saúde, UFSC/NuPaf, Editor. 1997: Florianópolis - SC.

7. Anais do 2 Congresso Brasileiro de Atividade Física e Saúde, U.-. NuPAF, Editor. 1999: Florianópolis - SC. p. 191.

8. Anais do 3 Congresso Brasileiro de Atividade Física e Saúde, N. UFSC, Editor. 2003: Florianópolis - SC. p. 1-220.

9. Anais do 4 Congresso Brasileiro de Atividade Física e Saúde, N. UFSC, Editor. 2003: Florianópolis - SC. p. 1-220.

10. Anais do 5 Congresso Brasileiro de Atividade Física e Saúde, N. UFSC, Editor. 2005: Florianópolis - SC. p. 208.

11. Anais do 6 congresso Brasileiro de Atividade Física e Saúde, N. UFSC, Editor. 2007: Florianópolis - SC. p. 208.

12. Anais do 7 Congresso Brasileiro de Atividade Física e Saúde, N. UFSC, Editor. 2009: Porto de Galinhas - PE. p. 226.

13. Anais do IX Congresso Brasileiro de Atividade Física e Saúde. 2013: Curitiba - Pr. p. 304.

14. Anais do VIII Congresso De Atividade Física e Saúde. 2011: Gramado - RS.

15. Grimes DA, Schulz KF. An overview of clinical research: the lay of the land. Lancet. 2002;359(9300):57-61.

16. Florindo AA, Hallal PC. Epidemiologia da atividade física, São Paulo: Atheneu, 2011.

17. A inserção da subárea de Atividade Física e Saúde nos programas de pós-graduação em Educação Física no Brasil.Virgílio, Viana Ramires, Leonardo Augusto Becker, Ana Daniela Izoton de Sadovsky, Adriana Marchon Zago, Renata Moraes Bielemann e Paulo Henrique Guerra

18. A contribuição do Brasil para a pesquisa em atividade física e saúde no cenário internacional Bruna Gonçalves Cordeiro da Silva, Juan Pablo Rey-López, Fernando Pires Hartwig, Thiago Gonzalez Barbosa e Silva, Renata Moraes Bielemann e Carolina de Vargas Nunes Coll

19. Reis RS. Reflexões sobre o IX Congresso Brasileiro de Atividade Física e Saúde. Rev Bras Ativ Fís Saúde. 2013;18(5):521-22.

20. Sá TH, Leandro MGL, Borges MC, Claro RM. Velhos e novos males da saúde no Brasil: a evolução do país e de suas doenças. São Paulo, 2014. $3^{\text {a }}$ ed. (prelo),

ENDEREC O PARA

CORRESPONDÊNCIA

TAIS TAIANA SARABIA

Praça Tiradentes, 370, apt.1301-

Centro, Curitiba Paraná- PR. CEP:

80020-100. Telefone: (41) 9666-8021

E-mail: tais.1986ahotmail.com 